Collectif, Entre le cour et le diaphragme: (d)écrire les émotions dans la littérature narrative et scientifique du Moyen Âge, actes de colloque (Louvain-la-Neuve, 8-9 décembre 2016)

\title{
Brînduşa-Elena Grigoriu
}

\section{OpenEdition}

\section{Journals}

Édition électronique

URL : https://journals.openedition.org/ccm/7418

DOI : $10.4000 / \mathrm{ccm} .7418$

ISSN : 2119-1026

Éditeur

Centre d'études supérieures de civilisation médiévale/Université de Poitiers

Édition imprimée

Date de publication : 1 juin 2021

Pagination : 161-165

ISBN : 978-2-490783-09-0

ISSN : 0007-9731

Référence électronique

Brînduşa-Elena Grigoriu, « Collectif, Entre le cœur et le diaphragme : (d)écrire les émotions dans la littérature narrative et scientifique du Moyen Âge, actes de colloque (Louvain-la-Neuve,

8-9 décembre 2016) », Cahiers de civilisation médiévale [En ligne], 254 | 2021, mis en ligne le 01 juin 2021, consulté le 30 novembre 2022. URL : http://journals.openedition.org/ccm/7418 ; DOI : https:// doi.org/10.4000/ccm.7418

\section{(c) $(1) \odot$}

Creative Commons - Attribution - Pas d'Utilisation Commerciale - Pas de Modification 4.0 International - CC BY-NC-ND 4.0

https://creativecommons.org/licenses/by-nc-nd/4.0/ 
Entre le coeur et le diaphragme: (d)écrire les émotions dans la littérature narrative et scientifique du Moyen Âge, actes de colloque (Louvain-la-Neuve, 8-9 décembre 2016), Craig Alexander BAKER, Mattia CAvagnA et Grégory Clesse (dir.), Louvain-la-Neuve, Institut d'études médiévales de l'université catholique de Louvain (Publications de l'Institut d'études médiévales. Textes, études, congrès, 30), 2018.

Placé sous le signe d'un changement de paradigme épistémologique que les médiévistes saluent comme un véritable «affective turn » touchant tout particulièrement les sciences humaines et sociales, le volume codirigé par Craig Baker, Mattia Cavagna et Grégory Clesse rassemble les actes du colloque international accueilli les 8-9 décembre 2016 par l'Université catholique de Louvain et soutenu par l'Université libre de Bruxelles et le Fonds national de la recherche scientifique de Belgique; tous corpus confondus (fussent-ils latins ou vernaculaires), il se propose d'explorer la pertinence thématique et la portée structurante des émotions au cœur de la littérature narrative et scientifique de la Romania médiévale.

Dans le dessein d'interroger «l'entrelacement parfois subtil des traditions médicale et littéraire » comme phénomène global de la civilisation médiévale ( $\mathrm{p}$. X de la Présentation), les trois éditeurs de Belgique s'appuient sur l'Encyclopédire: formes de l'ambition encyclopédique dans l'Antiquité et au Moyen Âge d'Arnaud Zucker, vaste ouvrage collectif paru chez Brepols en 2013 (Collection d'études médiévales de Nice, 14), sous la direction du chercheur niçois, et proclament l'universalité de « l'ambition encyclopédique » au Moyen Âge. Fédérés par l'aspiration de « contribuer à l'enrichissement de notre compréhension de la manière dont le Moyen Âge a perçu, pensé et exprimé les émotions » (p. XI), un ensemble de chercheurs européens provenant de plusieurs universités et instituts occidentaux (français, suisses et italiens) et de plusieurs champs scientifiques (histoire de la littérature, de la théologie, de l'anthropologie et des sciences médiévales) explorent, en français aussi bien qu'en italien, les œuvres les plus influentes des
$\mathrm{XII}^{\mathrm{e}}-\mathrm{XV}^{\mathrm{e}} \mathrm{s}$., intégrant dans leur réflexion les travaux considérés comme les plus représentatifs. La bibliographie de référence couvre les années 1976-2017 (cf. p. XI-XIV de la Présentation), cruciales pour «les questions épistémologiques qui se posent lorsque la notion d'émotion est mobilisée dans l'étude de la société médiévale » (p. VIII), et compte 19 titres français, 16 anglo-saxons, 10 italiens et 1 espagnol, propres à nourrir le dialogue interculturel au sein de la médiévistique européenne.

L'étude inaugurale de Jean-Marie Fritz, Émotions sanguines? Portraits de sanguin dans les textes latins et vernaculaires, commence par jeter les bases humorales du cadre conceptuel des émotions médiévales : un « quadrillage de l'espace » remontant à l'Antiquité, mémorablement illustré dès le XII ${ }^{\mathrm{e}} \mathrm{s}$. (dans le manuscrit de Prüfening) et permettant à l'homme-microcosme de déployer le spectre caractériel humain de façon apparemment symétrique : d'un côté, par deux tempéraments humides pauvres en émotions (flegmatique et sanguin), et de l'autre, par deux tempéraments secs (mélancolique et colérique) émotionnellement riches (p. 2). C'est dans ce cadre théorique que le chercheur de l'Université de Bourgogne lance sa quête, interpellant le sang et la " transparence du sanguin » au nom d'une « réparation » due précisément à cette humeur souvent négligée, contre laquelle se définissent toutes les autres. La " réparation » commence par un excursus auprès des sources, de Galien à Isidore de Séville via un traité grec anonyme, De la Constitution de l'univers et de l'homme, et, sur le plan des traits associés au tempérament sanguin, de la niaiserie à la suavité en passant par l'hilarité. C'est avec le De temporum ratione de Bède que 
s'impose l'antinomie sanguin-mélancolique, avec ses connotations émotivement chargées (p. 3). Médecins, encyclopédistes et philosophes cultivent cette vision tout au long du Moyen Âge central, en faisant la part belle au sang par rapport aux humeurs peccantes; cependant, un glissement se prépare des émois de bon aloi associés à la courtoisie vers la carnalitas de la luxure. Si Hildegarde de Bingen voyait le type sanguin comme « celui qui maitrise sa libido », l'optique change dramatiquement à la fin du Moyen Âge, lorsque le Mesnagier de Paris lui attribue un penchant naturel pour la " joliveté » (légèreté, coquetterie), Placides et Timéo pour le désir et Le Livre des fais et bonnes meurs du sage roy Charles $V$ de Christine de Pizan pour les « esbatements » de la jeunesse (p. 8). Enfin, chez Arnaud de Villeneuve, au début du XIV ${ }^{\mathrm{e}}$ s., le sanguin devient, par « accident de l'âme » (notion héritée d'Aldebrandin de Sienne), l'« imbécile heureux » servant de faire-valoir aux héros atteints d'amor heroïcus, comme Gauvain face à Yvain ou à Perceval (p. 14). Fort de ces nuances et muances de la laetitia-stultitia, le périple de J. -M. Fritz s'achève sur une allusion à Dante, qui situe la Joie aux débuts paradisiaques de l'existence humaine, le sanguin plongeant alors, innocemment, au tourbillon des « fins dernières » et aux envolées de la « vision béatifique ».

Avec l'article de Claire Donnat-Aracil, c'est une «mystique des affects » qui prend corps : on assiste à la mobilisation de l'anthropologie humorale par l'épistémè religieuse. La chercheuse de Paris III Sorbonne Nouvelle dégage " la présence filigranée d'une pensée médicale » dans la Vie des Pères, où le spectre émotionnel de la jalousie spirituelle puise dans la théorie des humeurs. C'est la mélancolie qui est particulièrement focalisée, considérée à la fois comme une maladie de l'âme (lorsqu'elle est « furieuse ») et comme un don divin conduisant à la conversion (lorsqu'elle est une forme paulinienne de « tristesse selon Dieu »; cf. p. 15-21 et n. 27). Associés aux péchés d'acédie et de cruauté, les tempéraments mélancolique et colérique demandent tout particulièrement à être maîtrisés et transcendés; en revanche, pour accéder à la sainteté, les ermites seraient tenus d'œuvrer au développement d'un tempérament sanguin susceptible de leur conférer une « douçor » s'ouvrant à la grâce divine. Et l'a. de préciser opportunément : « La conversion n'est alors plus seulement une affaire spirituelle, mais aussi humorale, et le discours théologique sur la pénitence est tissé de références à la pensée médicale » (p. 25). En somme, la pensée cistercienne sous-tendant la première Vie des Pères prône la guérison des affects par celle des humeurs, ces dernières étant subordonnées à l'exigence d'un dépassement (larmoyant) du corps, dans l'espoir d'une « reconstruction communautaire » reposant sur la « compassio ». Les rivalités se résolvent ainsi en liens communautaires, amicaux et empathiques. La question se pose alors de soupeser le degré de pertinence de cette forme humorale de pénitence pour d'autres textes relevant de la matière narrative vernaculaire : l'étude de C. Donnat-Aracil permet d'espérer que le dialogue médico-théologal imprégnant son corpus n'est pas un simple hapax francophone.

Avec Mattia Cipriani, de l'IRHT, l'analyse de l'incidence humorale sur le traitement narratif des émotions se poursuit en italien. Le Liber de natura rerum de Thomas de Cantimpré, de par sa vocation pratique, devient un champ où les affectus animi sont rarement nommés et encore plus rarement spécifiés (p. 38-39). Afin de cerner scientifiquement l'ampleur de ce phénomène elliptique par ailleurs compatible avec le profil d' " un opera pratico-naturalistica come il $L d n r$ ", le chercheur réalise un décompte des occurrences des émotions basiques amor, affectus; gaudium, laetitia, hilaritas; concupiscentia, libido, ardor, luxuria, appetitus; tristitia, egritudo, dolor, melancholia, desperatio; invidia; ira, iracundia et metus au sein du corpus. Trois points de vue sur les états d'âme sont ensuite explorés, donnant lieu à un encadrement allégorique, physiologique et psychologique, à un référencement attentif des sources consultées par le Dominicain et à une appréciation de sa contribution originale, notamment dans la saisie de la «diffusion de la luxure » (p. 43). Dans sa réflexion sur les passions, Thomas de Cantimpré tâcherait ainsi de concilier des acquis devenus classiques (remontant à la théologie augustinienne) avec les exigences du contexte moderne qui était le sien (sous l'influence grandissante d'Aristote et des modi pensandi relevant de la chirurgie salernitaine et de la praxis encyclopédique). La conclusion de cette étude abondamment documentée contribue à rehausser le rôle de l'encyclopédiste flamand dans le déploiement d'un « sottile momento di sintesi della cultura dell'Europa medievale », appréhendé dans son champ originairement conventuel. 
Dans sa quête des émotions positives et de leur topographie scientifique au XIII ${ }^{\mathrm{e}}$ s., l'étude d'Isabelle Draelants replace " la science de l'âme » dans son cadre épistémologique médiéval, qui « couvre à la fois l'étude de l'animation de tous les êtres vivants, du végétal au rationnel à en passant par l'animal » (p. 50). Si les spécialistes des « passions de l'âme » consacrent des traités à vocation encyclopédique à ce champ que la psychologie embrasse aujourd'hui de façon plus resserrée, c'est que les « sciences du vivant » cherchaient dès l'Antiquité un locus pour situer " le siège des sensations" en poursuivant, dès Homère et Galien, un modèle qui s'appuie, au Moyen Âge central, sur le « principe d'animation réparti selon les règnes naturels » (p. 54). La vision la plus répandue du gouvernement du corps doit beaucoup aux trois pneumata, respectivement « l'esprit animal dans le cerveau, l'esprit vital dans le cœur, l'esprit naturel ou physique dans le foie » (p. 55). Pour la chercheuse du CNRS-IRHT, le corpus offert par les Questions sur les animaux d'Albert le Grand se révèle « exploitable à propos des émotions » à la lumière du commentaire De animalibus, que le théologien et philosophe dominicain consacre à la conception naturaliste d'Aristote via la glose d'Avicenne et l'adaptation de Michel Scot. Avec le foie, c'est le cour qu'Albert définit comme foyer central des affects humains, tandis que le diaphragme remplit le rôle de " séparateur et discriminateur entre des parties anatomiques de natures différentes, mais aussi comme générateur et modérateur des émotions » (p. 69); si le cœur est le prince du royaume, le diaphragme devient sa « fortification » (p. 71). Le rire - réputé être l'apanage de l'homme - fournit un cas de figure intéressant, grâce au « titillement chaleureux » du diaphragme censé le déclencher; en outre, toute émotion, lorsqu'elle est appréhendée par Albert le Grand, est à mettre en rapport avec la physionomie, la morale pratique et l'intellect, dans ce que l'a. parisienne voit comme une « anthropologie fondée sur une continuité entre l'humain et l'animal » (p. 78).

Après le rire, ce sont la honte et la peur qui appellent l'attention des chercheurs : pour Amandine Mussou, de l'Université Paris Diderot - Paris 7, ces émotions choisissent, pour s'incarner, deux animaux totems, la licorne et le lièvre, et un corpus allant du Roman de la Rose de Guillaume de Lorris aux Eschés amoureux (en vers et prose) et au Livre des problèmes
d'Évrart de Conty. En outre, il s'agit d'émotions à vocation normative que les jeunes filles sont tenues d'éprouver et d'exprimer " pour se conduire convenablement » (p. 97). Les hommes, eux, se placent sous la bannière de Hardement, si bien que l'encodage allégorique des affects comporte une dimension genrée bien évidente dans les deux corpus narratifs, où le déplacement des pièces fait écho aux réactions physiologiques qui leur sont traditionnellement assignées. L'article réussit brillamment à rattacher les stéréotypes animaux aux manifestations romanesques et dramatiques des deux " passions » envisagées, inscrivant l'analyse dans la sociocritique des communautés émotionnelles, sans pour autant se rapporter à l'importante contribution de Barbara $\mathrm{H}$. Rosenwein (Emotional Communities in the Early Middle Ages, Ithaca/New York/Londres, Cornwell University Press, 2006; Generations of Feeling: A History of Emotions, 600-1700, Cambridge, Cambridge University Press, 2016) dans ce domaine si légitimement prometteur. Le médecin Évrart de Conty revient sous la plume de Béatrice Delaurenti, en tandem d'hilarité avec son contemporain théologien Nicole Oresme. Les deux « approches savantes du rire » viennent graver les années 1370-1380 et l'environnement affectif de la cour de Charles V dans l'histoire du « risus mediaevalis » projetée par Jacques le Goff (1989-1991) et développée par plusieurs groupes de recherche et chercheurs indépendants (mentionnés dans la note 4, p. 102). Le versant qui intéresse l'historienne de l'EHESS-CNRS est la question du « comment on expliquait le rire au Moyen Âge », abordée à partir des Problemata d'Oresme (édition sous presse chez les Belles Lettres, préparée sous la direction conjointe d'Alain Boureau et de Béatrice Delaurenti ellemême) et des Problemes d'Aristote d'Évrart de Conty. L'optique que partagent les deux naturalistes est de nature «psycho-physiologique », dans la mesure où elle embrasse « un processus interne dépendant de l'articulation de l'âme et du corps » (p. 109). En revanche, si Nicole Oresme distingue le rire complet du rire de chatouille (incomplet, car il ne dépend d'aucune émotion), son collègue médecin voit dans ce dernier une réussite salutaire du corps, consistant à «évacuer la tension provoquée par l'agression » (p. 112). Avec ou sans joie, le rire demeure, dans chacun de ces cas, une «dynamique corporelle et affective » (p. 116) plutôt qu'un moyen de communication humaine. 
Pour ce qui est de la communication littéraire vernaculaire, tout un volet d'études lui est consacré au sein du volume; le corpus narratif fictionnel comprend deux romans de Chrétien de Troyes : Yvain, scruté par Anatole Pierre Fuksas et Cligés, physiologiquement comparé par Giovanna Perrotta avec le Jaufre provençal; le Lancelot-Graal, où Camille Carnaille puise ses exemples les plus pittoresques de contrôle émotionnel; les adaptations d'Ovide en vers et en prose, explorées par Gioia Paradisi sous l'angle des émotions de l'éros, enfin le Breviari d'amor de Matfre Ermengaud, déployant, avec Guillaume Oriol, les lignes de force de la «palette émotionnelle» des troubadours.

Tout commence sous le signe du duo "vérité/ authenticité », qu'A. P. Fuksas mobilise pour aborder la question de la « folie » comme « mançonge », en faisant vibrer la corde intertextuelle de la fiction avec Wace et Geoffroy de Monmouth. À la lumière de la véracité générique, le chercheur de l'Université de Cassino crayonne une « vérité du roman » reposant sur la vérité des émotions décrites. Or, dans cette description, c'est l'idéal d'embellir la matière qui est poursuivie, le Tristan de Thomas révélant des affinités avec l'affabulation à ambition esthétique du Roman de Brut. Le topos de la sincérité de l'amour, si sensible dans le corpus tristanien, se retrouve chez Chrétien, qui fait usage du verbe " santir » tantôt dans son acception somatique, tantôt dans celle catégorielle. Le «san » romanesque n'exclut pas la théorie des humeurs, indirectement employée dans « les modulations affectives des personnages » (p. 129).

G. Perrotta aborde à son tour la "phénoménologie de l'amour » (p. 135) romanesque dans une démarche comparative où le dulce malum ovidien s'insère dans la trame narrative par le biais d'une imagerie médicale où les états d'âme sont traités sous un jour dysphorique et monologique. L'usage des contrefactuels à visée thanatique dans le langage amoureux singularise Jaufre par rapport au Cligés de Chrétien, et la médiéviste de Cassino avance l'hypothèse surprenante que le roman français n'aurait pas un équivalent lexical pour la drudaria provençale (la druerie étant effectivement absente au niveau textuel, tandis que le désir d'être-ensemble s'insinue malgré tout dans le flux verbal, aux vers 567, 790, 798 , etc.). Le dédoublement, le débat, la nuit comme « refuge émotionnel» sont des angles remarquablement enrichissants, que l'article aiguise en croisant, implicitement et puissamment, des outils conceptuels comme le régime nocturne de Gilbert Durand et le régime émotionnel de William $\mathrm{M}$. Reddy dans la saisie de la « vie littéraire du personnage » (p. 151). C. Carnaille, de l'Université de Genève, suit le cheminement tortueux de la colère et de sa maîtrise (la « garde », selon Brunetto Latini) au Moyen Âge central, en prenant Dieu comme point d'appui, les théoriciens médiévaux comme maîtres à penser, la furor épique et la courtoisie romanesque comme terrains d'expérimentation. Les fruits de cette lecture sont d'autant plus enrichissants qu'ils vont à contre-courant des horizons d'attente tracés pour les genres littéraires considérés. Ainsi, le principe de mesure rapproche des chevaliers comme Lionel, Lancelot, Gauvain d'un côté, et Raoul de Cambrai, de l'autre, l'accès à l'héroïsme mûr se faisant dans les deux corpus par le dépassement de la fougue juvénile, qui n'est tolérée qu'à titre de réservoir pulsionnel promettant de grandes prouesses, et non pour son caractère « desreez ».

L'article de G. Paradisi se focalise sur la « construction culturelle des émotions », et analyse les remaniements médiévaux de l'art d'aimer ovidien dans trois textes français du XIII' $\mathrm{s}$. : les versions en vers de maître Élie et de Jakes d'Amiens, et la traduction en prose réalisée par deux auteurs anonymes. La sensibilité à la modélisation genrée du profil de l'amant(e), la réévaluation de la dichotomie ratio-impetus et de ses avatars francophones, l'ancrage solide de l'amor vulnus dans son contexte d'émergence ovidien, ainsi que la considération des destinataires textuels et sociétaux, sont des qualités qui recommandent fortement cette exploration de la translatio française de l'Ars amatoria, malgré la narrativité plutôt normative qu'effective du corpus proposé par la chercheuse de l'Université de Rome « La Sapienza ». Sa contribution s'avère précieuse pour l'histoire des affects, soulignant pertinemment l'impact durable et (dé) formateur du texte ovidien sur « les masses » et les « maîtres », et circonvenant le développement de la misogynie médiévale.

Les troubadours ne pouvaient manquer de cette galerie émotionnelle où la description a droit de cité autant que la narration; l'étude de Guillaume Oriol, de l'Université Bordeaux Montaigne, enracine le «principe d'amour » aux sèves structurales de la Dame de l'Arbre, dans le respect du programme iconographique et textuel que met en œuvre le Breviari 
d'amor. De la «transcendance de principe » de l'amor de lonh à la suprême vertu du mariage, les émotions investies par Matfre Ermengaud se nourrissent de citations troubadouresques " hautement performatives » (p. 198) qui opèrent le montage véridique (et véridictionnel) d'une « dialectique de l'amour » à caractère « juridique et judiciaire » (p. 200 et 202). Une thérapie émotionnelle est à l'œuvre dans cette concertation polyvocale où les prises de position des troubadours remplissent une fonction testimoniale, constituant en même temps un tremplin paradoxal pour l'éthique conjugale. Ce dernier article tâche de rétablir la « dignité philosophique » de la culture médiévale de l'amour moyennant, contre et au-delà des troubadours.
Voguant à la recherche d'un lieu d'être « entre le cœur et le diaphragme » (en passant par le foie et quelquefois par les poumons), les émotions qui se déploient dans ce riche volume interpellent par leur représentativité des foyers énonciatifs médiévaux, intriguent par leur foisonnement descriptif et divertissent par leur portée intergénérique, interculturelle et intersémiologique. Elles se recommandent au lecteur désireux de connaître le profil affectif d'une culture dans son altérité sentie, sondée et reconstituée.

Brînduşa-Elena GrigoriU Université de Laşi (Roumanie) 